\title{
Telemetry System Based on Inductive-Coupling Transmission for Implantable Pressure Sensors: A Stent-Graft Endoleakage Monitor
}

\author{
Cristina Oliveira *, Nuno Almeida ${ }^{\ddagger}$ and José Machado da Silva * \\ *INESC TEC, Faculdade de Engenharia, Universidade do Porto, Portugal \\ Email: \{cristina.oliveira,jms\} @ fe.up.pt \\ ${ }^{\ddagger}$ Faculdade de Engenharia, Universidade do Porto, Portugal \\ Email: nunocmalmeida@gmail.com
}

\begin{abstract}
A direct arterial pressure monitoring system for post EVAR procedure based in inductive-coupling is presented which avoids the need to resorting to more complex biomedical imaging systems. Post stent-graft implantation complications, such as endoleaks, are detected with capacitive pressure sensors placed in the stent-graft and monitored externally after the measurement of the oscillation frequency of the $L C$ resonant circuit created by sensors and inductive coupling. Several measurements were performed in order to characterize the system and the results were compared with the expected values based on theoretical models. The first set of measurements were carried out for the purpose of testing the system's frequency detection accuracy along the working bandwidth and for varying distances between the reader and the sensors. For these measurements the air was used as the dielectric medium between the reader and the sensors antennas, and the detected oscillation frequencies were in accordance with the theoretical values. Also the performed measurements proved that the system is capable of correctly detect the oscillation frequencies of a cluster of sensors. In order to better characterize the system in accordance with its final application, the measurements were repeated for a dielectric medium with electrical characteristics similar to the human body (phantom).
\end{abstract}

Index Terms-inductive-coupling, stent-graft, endoleak, pressure sensors.

THE abdominal endovascular aneurysm repair (EVAR) is 1 a less invasive treatment for the repair of abdominal aortic aneurysms (AAAs) compared to the current standard procedure based on open surgery. Still, some problems, such as, endoleaks, graft migration, stent fracture, and enlargement of the aneurysm sac may occur which may require new surgery interventions. A regular monitoring/surveillance and behavioral analysis is thus needed to avoid the rupture of AAAs and to prevent re-interventions [1].

The pressure on the walls and in the aneurysm sac is one of the most important indicators for supervising post-EVAR patients. Direct intrasac pressure measurement outperforms imaging techniques in ascertaining either the complete AAA exclusion or the need for future interventions [2].

The main specifications of the pressure sensor required for aortic aneurysm pressure measurements [3] are: dynamic range between $20 \mathrm{mmHg}$ and $250 \mathrm{mmHg}$; a $1 \mathrm{mmHg}$ resolution; and an absolute accuracy below $5 \mathrm{mmHg}$.
Remote pressure monitoring systems [4] and results of clinical observation using these systems have been published [5], which show that the use of wirelessly accessed pressure sensors provide useful post-EVAR endovascular surveillance and help guiding clinical therapy.

Until now two remote pressure sensors have been tested in clinical trials, the EndoSure Sensor (CardioMEMS, Atlanta, GA, USA) and the ImPressure Sensor (Remon Medical Technologies, Caesarea, Israel) [6]. The ImPressure sensor is activated by ultrasound waves, which requires the use of an ultrasonic gel and direct contact between the skin and the transducer. Also the impossibility of ultrasound to travel through air or bone may lead to communication difficulties with the aneurysm sac. The APEX trial demonstrated the efficacy of the EndoSure sensor, a system that consists of a simple resonant circuit and a sophisticated external antennae. This sensor is delivered into the aneurysm sac through its own sheath, which adds more complexity to the EVAR procedure, and is completely separate from the aortic endograft so there is no control on the position of the sensor and is not possible to have more than one sensor in a patient due to the space a sensor occupies [4].

It is claimed that low frequency inductive-coupling based powering is limited in performance and applicability due to the need for low proximity and proper alignment between coupled coils.

\section{TElemetry System}

The alternative being proposed here allows deploying a cluster of sensors already integrated in the stent-graft and their monitoring using the same frequency range for powering and communication. The deployment of a cluster of sensors allows obtaining a more accurate observation of the pressure distribution in the aneurysm sac and improves reliability as more than one measurement are obtained simultaneously.

\section{A. Pressure Sensor}

Integrating the pressure sensors within the stent is possible because the pressure capacitive sensors being proposed for the system under development can be folded and collapsed 
together with the stent-graft without sacrificing its structure. This flexible capacitive sensor is fabricated using aligned carbon nanotubes (ACNTs) embedded in a flexible substrate of polydimethylsiloxane (PDMS), a transparent, non-toxic and biocompatible silicone elastomer.

Each sensing capacitor is actually attached to an inductor forming together an LC resonant circuit, whose oscillation frequency is sensitive to pressure variations, being each one allocated to a specific frequency in the $12.5 \mathrm{MHz}$ to $20.0 \mathrm{MHz}$ frequency range. An external reader delivers energy and detects the different resonance frequencies through an inductivecoupling link [7]. The $12.5 \mathrm{MHz}$ to $20.0 \mathrm{MHz}$ frequency band used here is specifically allocated for use in industrial, medical and scientific applications [8]. This guarantees some protection against biological effects and interferences generated by other electronic equipment and appliances. This frequency band is also the one where the minimum of total power loss due to skin-air interface and biological tissues absorption occurs.

Figure 1 shows the measured sensor capacitance versus pressure transfer characteristic. It can be seen that this characteristic shows relatively good linearity and fits very well that predicted by the analytical model. The pressures to be measured have been documented to be in the range of 40 $\mathrm{mmHg}(\sim 5.3 \mathrm{kPa})$ for the diastolic pressure and $170 \mathrm{mmHg}$ $(\sim 22.7 \mathrm{kPa})$ for the systolic pressure [9].

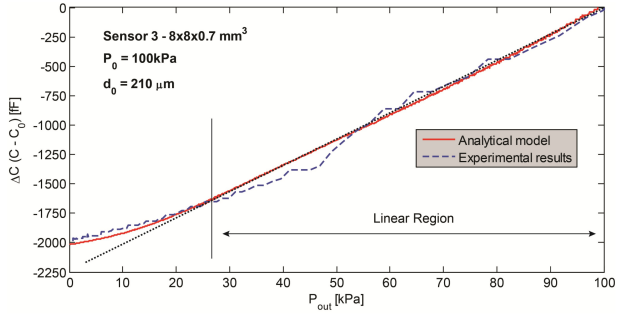

Figure 1: Sensor Pressure.

\section{B. External Reading System}

The external reading system (figure 2) includes a receiver capable of energizing the passive pressure sensors and detecting the sensors oscillation frequency, and a signal conditioning block composed of a transformer, amplifiers and a filter.

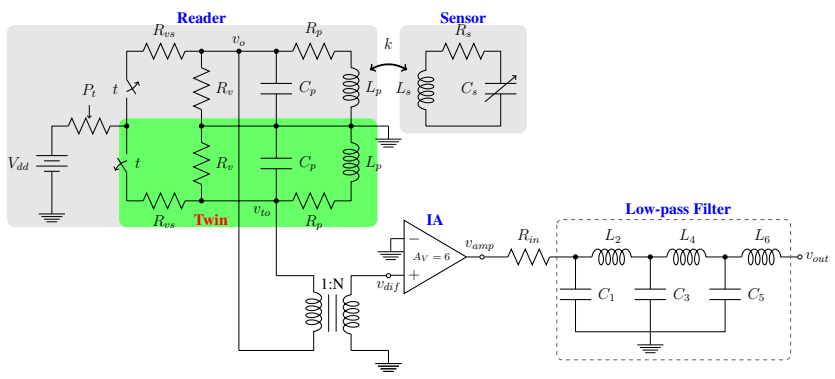

Figure 2: Schematic of the reader circuit.

To simplify circuit's analysis, only one sensor is by now used in the model (see figure 2). Its components can be modelled as passive elements: a variable capacitor $C_{s}$, whose value changes with the applied pressure, connected to an inductor $L_{s}$, implementing a parallel resonant circuit. The developed circuit resorts to a twin circuit added to the first part of the reader in order to provide a differential detection of the reflected oscillation. The sensor's oscillation frequency signal $v_{d i f}$ is obtained by subtracting the two signals in the receiver $v_{o}$ and $v_{t o}$ using a transformer.

The oscillation frequency (equation 1) is actually determined by the sensor's capacitance and inductance, as well as the coupling factor $k$ which depends on the reader's coil radius $r_{r}$, the sensor's coil radius $r_{s}$ and the distance between the antennas $x$, as depicted in equation 2 [10].

$$
\begin{gathered}
f_{\text {osc }}=\frac{1}{2 \pi \sqrt{\left(1-k^{2}\right) L_{s} C_{s}}} \\
k=\frac{r_{s}^{2} r_{r}^{2}}{\sqrt{r_{s} r_{r}}\left(\sqrt{x^{2}+r_{r}^{2}}\right)^{3}}
\end{gathered}
$$

Typically the potential abdominal aortic aneurysm stentgraft candidates are taken pre-procedural imaging exams consisting of both CT and catheter angiography, and for those who are submitted to an EVAR procedure imaging guidance is used to make sure the device is correctly placed and deployed [11]. With the data from these imagiological exams the exact positions and distances of the aorta, the aneurysm sac and the stent-graft are known, and therefore the $k$ can be estimated with reduced error.

\section{RESULTS}

For experimental evaluation purposes a prototype receiver circuit comprising an instrumentation amplifier and filtering was implemented. Circular copper wire-wound planar inductors and a ceramic capacitor were attached to form an $L C$ network, in order to emulate the sensor's behaviour. This section reports the obtained system characterization results in terms of the frequency detection accuracy in the presence of one or more sensors, at varying distances between the reader and the sensor(s).

\section{A. Frequency detection accuracy}

Measurements were obtained with different capacitors in the $L C$ network while maintaining the inductance constant $\left(L_{s}=5.42 \mu \mathrm{H}\right)$, and the results compared with the expected theoretical values given by equation 1 . Table I presents the measured and expected oscillation frequencies for a $10 \mathrm{~mm}$ distance between the reader and the sensor antennas. The $k$ was estimated using equation 2 for the expected oscillation frequency, and the obtained relative measurement error is below $4 \%$. The analogue to digital conversion and the Fast Fourier Transform (FFT) techniques used to capture and process the oscillation signal enable to obtain a $30.518 \mathrm{kHz}$ frequency detection resolution.

These measurements were repeated for distances of $20 \mathrm{~mm}$ and $30 \mathrm{~mm}$ and compared with the expected oscillation frequency, updating the $k$ value in accordance with the distance. The average relative measurement error was $2.2 \%$. 
Table I: Experimental and expected values of the oscillation frequencies detected for different sensor capacitances at a $10 \mathrm{~mm}$ distance.

\begin{tabular}{c|c|c|c}
\hline$C_{s}(\mathrm{pF})$ & $f_{\text {measured }}(\mathrm{MHz})$ & $f_{\text {expected }}(\mathrm{MHz})$ & Error $(\%)$ \\
\hline \hline 12 & 20.7 & 20 & 3.8 \\
15 & 18.6 & 18.2 & 2 \\
18 & 17.0 & 16.8 & 1.3 \\
22 & 15.4 & 15.0 & 2.6 \\
27 & 13.9 & 13.6 & 2.2 \\
33 & 12.6 & 12.7 & 1.4 \\
\hline
\end{tabular}

Table II: $L C$ pairs used for the measurements with air and a tissue emulating solution.

\begin{tabular}{c|c|c|c}
\hline Sensor & $L_{s}(\mu \mathrm{H})$ & \multicolumn{1}{c}{$C_{s}(\mathrm{pF})$} & $f_{\text {osc }}$ \\
\hline \hline 1 & 5.42 & 27 & 13.16 \\
2 & 3.85 & 22 & 17.29 \\
3 & 3.83 & 18 & 19.17 \\
\hline
\end{tabular}

\section{B. Effect of biological tissue in the detection}

Since the final application of the system is to read from sensors implanted inside the human body, further experiments were performed in order to assess the behaviour of the system in the presence of biological tissue between the antenna and the sensor.

Three sensors, each with a different oscillation frequency, were used to evaluate system's performance in presence of different transmission media (air and a tissue emulating solution). The sensors' natural oscillation frequencies were estimated using equation 3 , and the values of the inductance and capacitance were chosen in order to provide working frequencies in the specified range $(12.5 \mathrm{MHz}-20 \mathrm{MHz})$. Table II displays the characteristics of the $L C$ pairs used in the measurements.

$$
f_{\text {osc }}=\frac{1}{2 \pi \sqrt{L_{s} C_{s}}}
$$

A tissue emulating solution (phantom) with electrical characteristics (conductivity and permittivity) similar to the muscle tissue made according the recipes developed by [12]. First measurements using air as the medium between the reader and the sensor antennas were carried-out for different distances ranging from $5 \mathrm{~mm}$ to $50 \mathrm{~mm}$, in steps of $5 \mathrm{~mm}$, in order to have a comparison.

The emulating solution was poured in a beaker in order to achieve the desired height, corresponding to the distance between the reader and the sensor. The reader antenna was placed under the beaker and the sensor antenna was inserted in a hermetically sealed bag for protection and laid on top of the solution.

As can be observed in figures 3, 4, 5, the presence of the tissue causes a significant shifting in the received signal frequency. This can be due to the change in the medium permittivity to magnetic induction, also affected by the quality factor, as has been reported at [13], [14].

Regardless of this frequency shifting, when comparing the signals in the presence and absence of the phantom, the detected signals showed signal-to-noise and amplitude characteristics that allowed an easy detection of the sensor's resonant frequency, in the different cases.

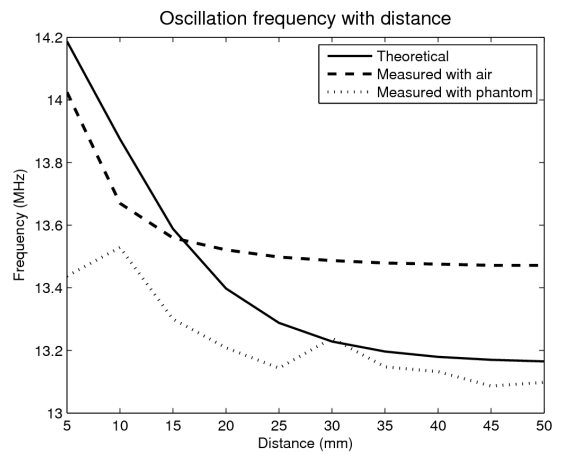

Figure 3: Oscillation frequencies (theoretical, measured in the presence of air and measured in the presence of the phantom) over a distance range for sensor 1 .

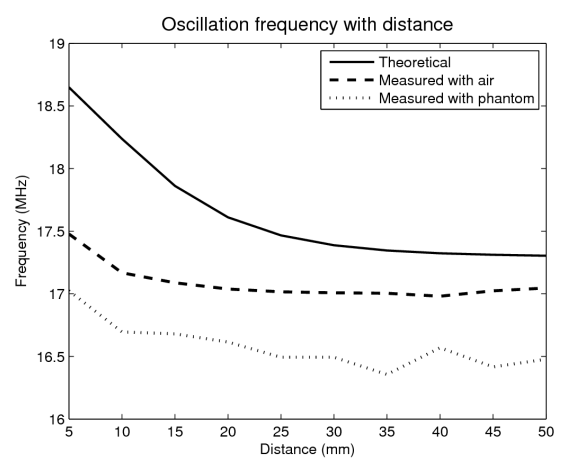

Figure 4: Oscillation frequencies (theoretical, measured in the presence of air and measured in the presence of the phantom) over a distance range for sensor 2 .

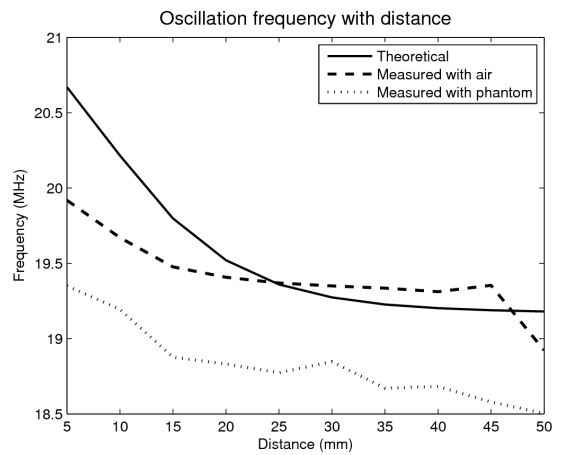

Figure 5: Oscillation frequencies (theoretical, measured in the presence of air and measured in the presence of the phantom) over a distance range for sensor 3 .

Although the calculated and measured oscillation frequency curves follow the same tendency, the equation 1 used to estimate the oscillation frequency (considering air as the dielectric) is clearly insufficient to predict the behaviour of the system specially in the presence of biological tissue. A path 
loss (PL) model would help to design optimal communication between the sensors and the reading system. One has to consider also that the setup used for the measurements was unable to guarantee an accurate distance and a perfect alignment between the antennas which could influence the results. The measurements were carried out in a heterogeneous medium, but the thoracic wall may have different tissues and tissue-air interfaces between the aorta and the skin surface, and therefore a more complex phantom is needed to fully characterize the system for biomedical applications.

\section{Detecting multiple sensors}

In order to study the functioning of the system with a cluster of sensors, a set of measurements with two different sensors placed in front of the reader's antenna was performed. The sensors characteristics (inductance and capacitance) are displayed in table III, as well as the calculated and measured oscillation frequencies for a distance of $10 \mathrm{~mm}$ between the sensors and the reader.

Table III: Sensors used for the measurements with air and tissue emulating solution.

\begin{tabular}{c|c|c|c|c}
\hline Sensor & $L_{s}(\mu \mathrm{H})$ & $C_{s}(\mathrm{pF})$ & $f_{\text {calc }}$ & $f_{\text {meas }}$ \\
\hline \hline 1 & 5.42 & 22 & 15.1 & 14.7 \\
2 & 3.85 & 27 & 16.2 & 16.2 \\
\hline
\end{tabular}

The obtained FFT spectra allow us to clearly distinguish the two frequencies, provided these are conveniently separated, as it is shown in figure 6 . Taking the frequency difference obtained here as the width of the different sub-bands allocated in the $12.5 \mathrm{MHz}$ to $20.0 \mathrm{MHz}$ range, a cluster of five sensors could be used. In order to ensure the required $230 \mathrm{mmHg}$ pressure measurement resolution in each sub-band a frequency detection resolution of $6.5 \mathrm{kHz}$ is needed. A resolution of 3.8 $\mathrm{kHz}$ could be obtained after improving the FFT algorithm and maintaining the same ADC sampling frequency.

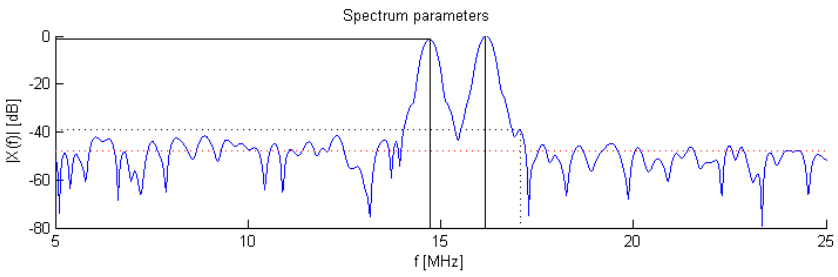

Figure 6: Receiver circuit output in the presence of two sensors.

\section{CONCLUSIONS AND Future WORK}

A telemetry system for wireless monitoring of coronary stent-grafts condition relying on capacitive pressure sensors attached to the stent is being developed. Experimental results validate the system's operation with the air as the dielectric medium between the reader and the systems antennas. Nevertheless a more complex model for the estimation of the oscillation frequency needs to be developed in order to know the relative frequency shift caused by the presence of biological tissue between the reader and the sensors.

\section{ACKNOWLEDGMENTS}

This work is financed by National Funds through the FCT - Fundação para a Ciência e a Tecnologia (Portuguese Foundation for Science and Technology) within programme MIT|Portugal, grant MIT-Pt/EDAM-EMD/0007/2008 and grant contract SFRH/BD/81476/2011.

\section{REFERENCES}

[1] B. Katzen and A. MacLean, "Complications of endovascular repair of abdominal aortic aneurysms: A review," CardioVascular and Interventional Radiology, vol. 29, pp. 935-946, 2006.

[2] B. Sonesson, N. Dias, M. Malina, P. Olofsson, D. Griffin, B. Lindblad, and K. Ivancev, "Intra-aneurysm pressure measurements in successfully excluded abdominal aortic aneurysm after endovascular repair,' Journal of Vascular Surgery, vol. 37, no. 4, pp. 733 - 738, 2003.

[3] J. Potkay, "Long term, implantable blood pressure monitoring systems," Biomedical Microdevices, vol. 10, pp. 379-392, 2008.

[4] F. Springer, R. W. Günther, and T. Schmitz-Rode, "Aneurysm Sac Pressure Measurement with Minimally Invasive Implantable Pressure Sensors: An Alternative to Current Surveillance Regimes after EVAR?" Cardiovasc Intervent Radiol, vol. 31, no. 3, pp. 460-467, 2008.

[5] C. J. Parsa, M. A. Daneshmand, B. Lima, K. Balsara, R. L. McCann, and G. C. Hughes, "Utility of remote wireless pressure sensing for endovascular leak detection after endovascular thoracic aneurysm repair," The Annals of Thoracic Surgery, vol. 89, no. 2, pp. 446 - 452, 2010.

[6] R. Milner, K. Kasirajan, and E. L. Chaikof, "Future of endograft surveillance," Seminars in Vascular Surgery, vol. 19, no. 2, pp. 75 82, 2006.

[7] C. Oliveira, N. Almeida, and J. Machado da Silva, "Inductive coupling system for endovascular aneurysm repair monitoring," Studies in health technology and informatics, vol. 177, pp. 101-6, 2012.

[8] "The European table of frequency allocations and utilizations in the frequency range $9 \mathrm{KHz}$ to $3000 \mathrm{GHz}$," Electronic Communications Commitee (ECC), Tech. Rep., 2009.

[9] T. Ohki, K. Ouriel, P. G. Silveira, B. Katzen, R. White, F. Criado, and E. Diethrich, "Initial results of wireless pressure sensing for endovascular aneurysm repair: The apex trial-acute pressure measurement to confirm aneurysm sac exclusion," Journal of Vascular Surgery, vol. 45, no. 2, pp. 236-242, 2007.

[10] K. Finkenzeller, RFID Handbook: Fundamentals and Applications in Contactless Smart Cards and Identification, second edition ed. John Wiley \& Sons, 2003

[11] J. A. Kaufman, S. C. Geller, D. C. Brewster, C. M. Fan, R. P. Cambria, G. M. LaMuraglia, J. P. Gertler, W. M. Abbott, and A. C. Waltman, "Endovascular repair of abdominal aortic aneurysms current status and future directions," American Journal of Roentgenology, vol. 175, no. 2, pp. 289-302, 2000.

[12] G. Hartsgrove, A. Kraszewski, and A. Surowiec, "Simulated biological materials for electromagnetic radiation absorption studies," Bioelectromagnetics, vol. 8, no. 1, pp. 29-36, 1987.

[13] A. Ingelman-Sundberg and E. Odeblad, "Radio frequency absorption in tissues with special reference to diagnostic applications in gynaecology," BJOG: An International Journal of Obstetrics \& Gynaecology, vol. 72, no. 6, pp. 940-945, 1965.

[14] M. Yvanoff and J. Venkataraman, "A feasibility study of tissue characterization using LC sensors," Antennas and Propagation, IEEE Transactions on, vol. 57, no. 4, pp. 885 -893, april 2009. 